\title{
Protecting our Elders and Developing Equitable Policies During Public Health Emergencies: Ethical Recommendations
}

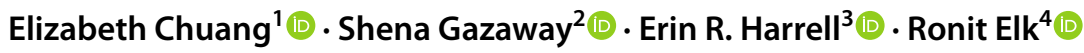

Accepted: 2 August 2021 / Published online: 5 November 2021

(c) The Author(s), under exclusive licence to Springer Science+Business Media, LLC, part of Springer Nature 2021

\begin{abstract}
Purpose of the Review Experiences of patients, families, healthcare workers and health systems during the COVID-19 pandemic and recent national focus on racial justice have forced a reconsideration of policies and processes of providing care in crisis situations when resources are scarce. The purpose of this review is to present recent developments in conceptualizing ethical crisis standards.

Recent findings Several recent papers have raised concerns that "objective" scarce resource allocation protocols will serve to exacerbate underlying social inequities. Older adults and their formal and informal caregivers suffered from intersecting planning failures including lack of adequate stockpiling of personal protective equipment, failure to protect essential workers, neglect of long-term care facilities and homecare in disaster planning and de-prioiritization in triage algorithms.

Summary Revision of disaster planning guidelines is urgent. The time is now to apply lessons learned from COVID-19 before another disaster occurs. We present several suggestions for future plans.
\end{abstract}

Keywords Triage $\cdot$ Health disparities $\cdot$ Ethics

\section{Introduction}

Public health ethics calls for a shift in focus from individual health to population health during public health disasters. The National Academy of Medicine (NAM) articulated an

Elizabeth Chuang

echuang@montefiore.org

Shena Gazaway

gazaways@uab.edu

Ronit Elk

relk@uabmc.edu

1 Department of Family and Social Medicine, Albert Einstein College of Medicine, 3347 Steuben Avenue, 2nd Floor, Bronx, NY 10467, USA

2 Department of Family, Community, and Health Systems, School of Nursing - University of Alabama Birmingham, 1720 2nd Avenue South, Birmingham, AL 35294, USA

3 Department of Psychology, College of Arts and Sciences, University of Alabama, Hackberry Lane, Box 870348, Tuscaloosa, AL 35487, USA

4 Division of Geriatrics, Gerontology and Palliative Care, Department of Medicine, School of Medicine University of Alabama, Birmingham, AL 35205, USA ethical framework for such times in 2012 which included the following features; (1) fairness, or prioritization based on relevant factors such as greater exposure to disease or community goals such as keeping key personnel at work, rather than "irrelevant" factors such as race, ethnicity or religious affiliation, (2) the duty of care, which states that those caring for individual patients should not simultaneously make decisions to benefit the group rather than the individual patient (i.e. separation of triage and care duties), (3) the duty to steward resources, which calls for withholding or withdrawing resources from patients who will not benefit from them, (4) transparency, (5) consistency, (6) proportionality, e.g. that burdens such as quarantine should be commensurate with the scale of the disaster and (7) accountability [1]. Protocols to triage scarce resources attempt to operationalize this framework; promoting fairness by reducing the impact of clinician biases, preserving the primary physician's duty to care by shifting some decisions to a triage team, and stewarding resources by creating algorithms aimed at maximizing lives saved [2]. The existence and publication of protocols promote transparency, consistency, and proportionality. Community engagement studies have identified normative values for triage, [3] and based on these normative values, many states have adopted triage protocols 
Table 1 Recent developments in triage literature:

\begin{tabular}{|c|c|c|}
\hline \multicolumn{2}{|l|}{ Category } & \multirow[t]{2}{*}{ INNOVATION } \\
\hline Title & Author, Year & \\
\hline $\begin{array}{l}\text { Respecting Disability Rights-Toward Improved Crisis } \\
\text { Standards of Care }\end{array}$ & Mello et al. $\mathbf{2 0 2 0}$ & $\begin{array}{l}\text { Removal of categorical exclusions that preclude groups } \\
\text { from accessing scarce life-sustaining resources }\end{array}$ \\
\hline $\begin{array}{l}\text { Eliminating Categorical Exclusion Criteria in Crisis Stand- } \\
\text { ards of Care Frameworks }\end{array}$ & Auriemma et al. $\mathbf{2 0 2 0}$ & $\begin{array}{l}\text { Removal of categorical exclusions that preclude groups } \\
\text { from accessing scarce life-sustaining resources }\end{array}$ \\
\hline $\begin{array}{l}\text { Mitigating Inequities and Saving Lives with ICU Triage } \\
\text { during the COVID-19 Pandemic }\end{array}$ & White and Lo 2020 & $\begin{array}{l}\text { Adding "equity weights" to give some priority to } \\
\text { disadvantaged groups, e.g. using the Area Deprivation } \\
\text { Index, prioritizing broadly-defined essential workers }\end{array}$ \\
\hline $\begin{array}{l}\text { Rationing, racism and justice: advancing the debate around } \\
\text { 'colourblind' COVID-19 ventilator allocation }\end{array}$ & Schmidt et al. 2021 & $\begin{array}{l}\text { Adding "equity weights" to give some priority to } \\
\text { disadvantaged groups, e.g. using the Area Depriva- } \\
\text { tion Index, considering adjusting or eliminating SOFA } \\
\text { score-based algorithms }\end{array}$ \\
\hline
\end{tabular}

to maximize short-term survival, which is achieved through use of scoring algorithms [4]. There is growing consensus that considerations of long-term survival, age, and quality of life are ethically problematic in triage decisions and tend to replicate social inequities $[5 \bullet, 6]$ (Table 1 ). Many protocols have moved away from such exclusions.

Recent developments in the field of triage ethics reflect lessons learned from the COVID -19 pandemic. This pandemic made it very clear that use of such triage algorithms would reinforce inequities existing outside of the hospital. People with pre-existing vulnerabilities to disease due to structural inequities would fare more poorly in these scoring systems and thus be required to relinquish their claims on resources if these protocols are used [7•]. While seeking to promote fairness, these triage policies have been agnostic to structural and systemic factors preceding the emergency, focusing narrowly on survivability. They are therefore silent on the unfairness resulting from structural racism. As such, these policies are an example of systemic racism, in which procedures and processes embedded in institutions perpetuate racial inequity [6]. In 2010, Ford and Airhihenbuwa articulated a framework to apply critical race theory to public health praxis [8]. They call for public health practice to be race-conscious by explicitly examining racism's potential contributions to inequities. They also call for centering on the margins, or shifting a discourse's starting point from a majority group's perspective to that of the marginalized group or groups. In this paper, we will use these concepts to evaluate the intersection of racism and ageism in the allocation of scarce resources during the COVID-19 pandemic, and to make recommendations for more ethical practice in future public health disasters.

\section{Allocation of Scarce Resources in Non-emergency Settings}

Several examples illustrate how racism and agism have been evident in scarce resource allocation in non-emergency settings even before the current focus on disaster triage. These flaws demonstrate that inherent biases are a part of the fabric and construction of guidelines which do not address or accommodate structural, environmental, and systemic racism and complex attitudes of ageism.

Although triage is an old concept in military contexts, allocation of scarce resources emerged as a theme in the general medical literature with the advent of hemodialysis to treat end stage kidney failure. Medical professionals needed a mechanism to allocate the limited number of dialysis machines to individual patients who were medically similar. An evaluation of the patient's "social worth" was used as the determining factor. Given that dialysis was only available initially at a single institution, the Admissions and Policies Committee of the Seattle Artificial Kidney Center at Swedish Hospital, also known as the "God Committee," was appointed to make these assessments. This committee of volunteers was selected as a representation of the larger society from a socioeconomic and occupational perspective, however, the majority of members were white and male. Medical professionals instructed them to exclude children and those over the age of 45 from consideration [9]. Using social worth as an ethical framework to allocate dialysis machines, the God Committee ultimately chose mostly white male patients to be recipients of this scarce life-saving resource, a clear illustration of how both agism and racism have always been intrinsic properties of triage systems. 
Modern ethical frameworks have moved away from explicitly factoring in "social worth" favoring a stance that honors all lives' inherent moral worth and recognizes that such determinations are subject to multiple biases and favor those who have more opportunities over their life course. Hemodialysis is no longer officially rationed in the United States; however undocumented immigrants are barred from obtaining insurance for this expensive medical resource. Therefore, they are de facto excluded from receiving ongoing dialysis.

Another example of modern-day rationing of scarce medical resources is in organ transplantation. There is a significant shortfall of available organs to treat all patients with vital organ failure [10]. Allocation decisions are made by organ donor networks; however individual transplant teams decide whether to list a patient as a potential recipient. To maximize the benefit of each organ, the age of organ is matched with age of recipient. While explicit references to social worth are not included in these decisions, teams deliberate on whether the transplant will be successful in the long term. In making this decision, teams consider whether the patient has "social support" to help them cope with chronic immunosuppressant treatment to maintain the transplant, and the patient must demonstrate evidence that they will comply with follow-up treatment [11]. These considerations tend to reproduce biases that disadvantage marginalized populations in transplant decisions, and there is little data to support their usefulness in predicting long-term success of the transplant [12].

Social properties embedded into how metrics are used to assign priority for seriously ill patients continue to create barriers for African American patients with kidney failure. One barrier is that some providers believe that African Americans are more content on dialysis than Whites [13, 14]. Second, African Americans from low socioeconomic backgrounds are more likely to see primary care providers who know less about transplantation [14]. Experiences of systemic and interpersonal racism may lead some patients to be warier about consenting to transplant, and providers may misinterpret patient indecision as lack of interest [14]. All of these concerns highlight flaws within structured, objective systems. Timely access to scarce resources is limited for marginalized groups even outside of public health emergencies. Thus, even as allocation procedures have evolved over time in attempts to improve fairness, disparities in access to scarce resources continue to be replicated over time.

\section{Allocation of Scarce Resources in Public Health Emergencies, Pre-COVID-19}

The tragedy of multiple deaths at Memorial Medical Center in New Orleans immediately after Hurricane Katrina illustrated an urgent need to develop standard protocols to ration scarce resources in disaster conditions when the standard of care cannot be provided to all $[15,16]$. At Memorial, the electrical generators flooded in the aftermath of the storm, and there was no electricity supplied to the hospital for several days. Temperatures reached 110 degrees inside the building. Evacuation of the approximately 2,000 patients, families, and staff had to occur via a helipad from a neighboring building. Patients were transported down the stairs of the hospital and back up to the helipad through a small opening. The hospital staff created an ad hoc triage system, giving patients the labels 1,2 , and 3 to order their evacuation, with 1 given the highest priority. Patients with do-notresuscitate (DNR) orders were de-prioritized systematically. One patient, in particular, Emmett Everett, an obese, paraplegic, African American patient, was in stable condition but allegedly given a lethal combination of drugs because the staff didn't think they would be able to evacuate him, making the decision based on his weight and perhaps their own implicit bias. These events highlighted the dangers of lacking a pre-specified protocol for triage and guidelines for withdrawing or withholding life-sustaining treatments. The ad hoc decisions made by the Memorial staff raise concerns about racism and ableism. De-prioritizing otherwise stable patients with DNR orders who are more likely to be elderly introduces systematic ageism.

Although triage guidelines have more recently narrowly focused on the likelihood of surviving the acute event to avoid systematic bias against marginalized populations and older individuals, $[2,6]$ the use of numeric scores may continue to replicate health disparities. Patients with poor health due to structural determinants of health are likely to become more ill compared with those who have access to healthy living environments in the face of a new disease such as COVID-19, resulting in marginalized groups receiving poorer scores and de-prioritization within these algorithms $[17,18,19 \bullet \bullet, 20]$. Some may argue that it is too late to rectify long-standing social inequities at the door of the Intensive Care Unit or Emergency Room when patients are in extremis; allocating a scarce resource to someone who will not survive will not improve equity. However, for this 
justification to be accurate, algorithms must correctly identify those who are imminently dying with or without the resource [7•]. Furthermore, although it may be too late to reverse the results of pre-disaster inequities, neither ought triage protocols worsen them.

The NAM acknowledged that when it may not be possible to give everyone the care needed to survive, difficult choices must be made to balance fairness and the duty to care with the duty to steward resources [1]. The framework points out that wasteful use of scarce resources results in excess mortality. While providing a resource to a patient who is imminently dying with or without the resource is clearly wasteful, it is less clear whether the use of triage algorithms to maximize lives saved is the same as avoiding wasteful care. Triage algorithms that use quantitative scores such as the Sequential Organ Failure Assessment (SOFA) score poorly distinguish between those who will and will not benefit from a particular resource [21, 22]. Providing a resource to an individual with a slightly or moderately lower probability of benefit than another individual with a slightly or moderately higher probability is not clearly wasteful, and the first individual may rightly have some claim to the resource [23].

There is evidence that people of all ages tend to justify prioritizing younger patients over older patients either by giving lower priority to older patients or by using age as a "tiebreaker" for otherwise similar patients. The justification provided for this is that a young person stands to lose more by losing the chance to live through life's stages if they are not given a life-sustaining resource, and therefore the young person has more claim to that resource (sometimes referred to as the "fair innings" argument) [24]. Age also may be considered a prognostic factor in algorithms seeking to prioritize those most likely to survive. However, the same issues arise as above if age does not completely predict survival. Some juristictions provide legal protections and do not allow age to be used in such protocols.

\section{Allocation of Personal Protective Equipment (PPE)}

Personal protective equipment (PPE), which can include gloves, face masks, face shields, goggles, respirators, and gowns, is essential in helping prevent the spread of infection to and from health care workers and patients. During the COVID-19 pandemic, health care workers faced a shortage of PPE while caring for patients presenting with a highly infectious and transmittable disease. When the pandemic was at its worst in 2020, news articles featured unconventional solutions of PPE, with health care workers using plastic garbage bags for gowns and plastic water bottle cutouts for eye protection [25]. Stories such as this made it clear that while PPE shortages certainly pose a problem for health care workers, it is not a problem that they have been trained to address or should be expected to solve [26]. Ethical guidelines published early in the pandemic specifically recommended considering all health care workers for protection, including those that work in transport, food, and environmental services [27]. However, racial minority health care workers, who are less likely to be represented among doctors and administrators highest in the workplace hierarchy, were most likely to contract COVID-19 [28].

Long-term care (LTC) facilities were hit hard by the pandemic. While LTC patients represented only about $6 \%$ of cases, they bore a strikingly disproportionate share of mortality at about $40 \%$ of total deaths [29]. In addition, clustering in nursing homes resulted in some facilities becoming overwhelmed with cases, particularly early on in the pandemic. The NAM Crisis Standards of Care report recognizes that "much of the disaster and surge planning focuses on hospitalized care (p. 5-1)." [1] However, very little space in the more than 500-page document is devoted to LTCs. When LTCs are mentioned it is in reference to their potential capacity to accommodate hospital discharges, noting that they "should not be overlooked as a resource." [1] Although the report recognizes that LTCs may be disproportionately impacted by certain incidents such as a novel influenza strain, it is clear that LTCs were not prioritized for distribution of PPE in the early phases of the pandemic [30]. In a survey, LTC professionals reported the emotional distress they experienced when COVID-19 hit with little preparation in place. These workers reported engaging in practices such as using garbage bags to protect themselves after having reused gowns, going in and out of rooms with the same gown, and using face masks for a week [31]. When PPE was sourced, the sourced PPE was often "regular" size, rather than a variety of sizes manufactured to fit the individuals who would need to use it, resulting in gender disparities [32]. For example, N95 masks must be fitted to provide an airtight seal to be protective. Many women require "small" sized masks for a proper fit.

Several factors converged for LTC professionals, including a general lack of PPE, PPE that does not meet the standard of protection to prevent transmissions, and greater risk of illness from contracting COVID-19 among marginalized groups. Lack of PPE may have exacerbated other vulnerabilities such as living in multigenerational homes. This placed many workers in an extreme circumstance of choosing to what extent they could balance risk of not protecting themselves while also providing care for others and financially supporting their families, including dependent elders [33]. It has been posited that this embodiment of risk is gendered and racialized, [33] and that those in charge of decision-making contributed to this inequity by placing frontline workers in environments that offered inadequate self-protection beyond what would be considered acceptable 
for occupational risk. Indeed, LTC facility workers represented the largest proportion of health care workers sickened by COVID-19 in the spring of 2020 [28].

\section{Mental Health Repercussions On Those Working Through Crisis}

Clinicians who perceive the racism and ageism built into the algorithms will experience moral distress in their implementation, making the mental health repercussions even more severe. Healthcare providers are at risk of experiencing burnout, distress, depression, and other mental health concerns related to their work's stressful and highly varied nature $[34,35]$. Before COVID-19, literature suggested multiple causes of this mental health vulnerability including scheduling, administrative work, and the nature of healthcare work - prioritizing others before self [34]. Now, when faced with COVID-19, additional triggers for a mental health illness emergency have been added - job insecurity, more extended periods of isolation, and increased psychological burden [36]. The need to allocate life-saving resources in times of scarcity is an additional psychological burden of the COVID-19 pandemic.

Kim et al. defines "the psychological burden of workers in terms of emotions such as concern, anxiety, uneasiness, and depression felt by workers due to their concerns about the success or failure of their work and their safety, which are affected mainly by the workload and work conditions" pg. 225 [37]. In times of pandemic or other rapid onset medical catastrophe, healthcare providers have the potential of being placed in uncomfortable and stressful situations as they begin to think about the resources they have and doing the greatest good for the most. Clinicians have for years relied on objective measures to help guide them during this ethical decision-making process. But as we have presented and literature supports, these objective measures at their core build upon an unjust and unequitable framework when assigning objective scores to individuals who have not had the same access to quality healthcare or living/ working environments throughout their lives and those living with disabilities $[6,38]$.

One goal of these guidelines, articulated in the "duty to care" component, is to remove the excessive burden of decision-making from the bedside clinician's shoulders. However, this does not take into consideration the increased potential psychological burden if these triage guidelines are not viewed by the medical professionals as fair [6]. Healthcare providers are charged to provide individualized care to the extent of their abilities. With the novel burden of triage added to the everyday stressors of working as a healthcare professional, we must better assist these professionals with their mental health.

\section{Conclusions and Recommendations}

While approaches to fair allocation of scarce medical resources in both routine and disaster conditions have evolved, we have demonstrated how they continue to replicate the systemic inequities in our society. Harms to vulnerable elders were compounded by multiple intersecting factors including failure to protect essential front-line workers' mental and physical health in LTCs, lack of PPE which was worse in LTCs, and triage policies that prioritize younger patients (Fig. 1). An unmeasured and potentially vast and enduring harm of this replication is that it may further erode the trust in medical institutions among marginalized individuals. The NAM framework features fairness, the duty of care, the duty to steward resources, transparency, consistency, proportionality, and accountability. Within this framework, we recommend redoubling the focus on the duty to care and narrowly defining the duty to steward resources as the first steps to mitigate these errors.

First, the duty to care must be preserved through several concrete actions that can be taken by governments and institutions. Definitions of essential workers for PPE distribution should be revisited, and acute care hospitals should not be prioritized over other healthcare settings such as LTCs or home health. Institutions should ensure the equitable distribution of PPE among affected staff. Local and regional governments should actively manage PPE stockpiles to ensure

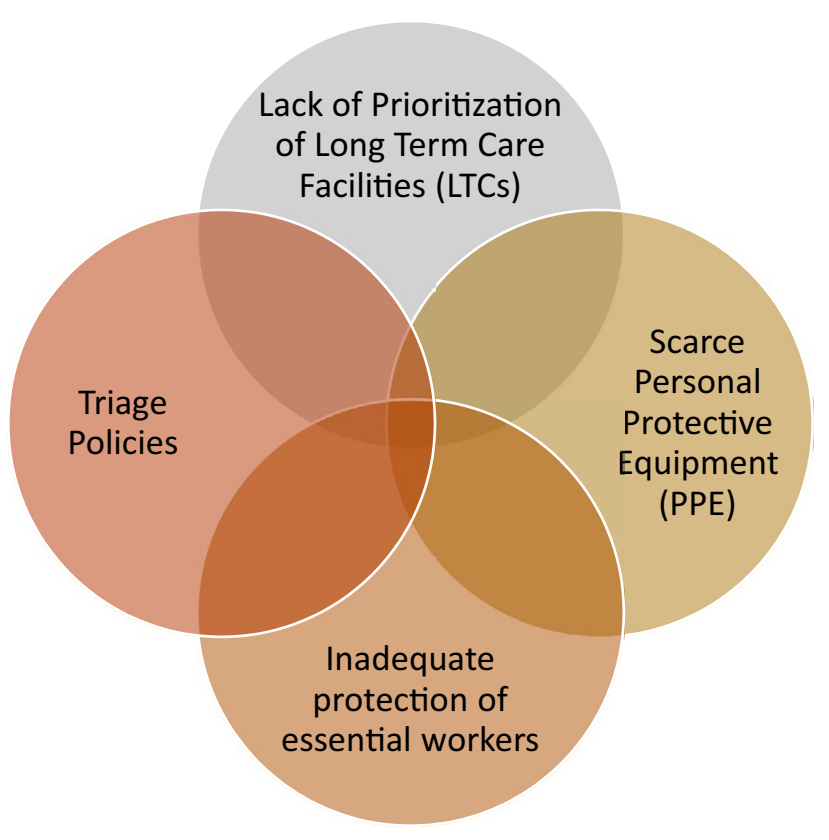

Fig. 1 Intersecting vulnerabilities of older adults in the pandemic 
that such shortages never occur again. Additionally, institutions and government agencies should work to build more redundancy in health care systems. Our health care industry does not invest in personnel, facility, or equipment surge capacity as building in redundancy is not a profitable line. Unfortunately, this orientation will result in the recurrence of periods of scarcity requiring rationing. Public perception of the healthcare system as a purely transactional enterprise is growing, along with a concerning level of distrust [39]. Governmental investment in redundancy proved inadequate in this crisis.

Second, the duty to steward resources should be narrowly defined to avoid allocating scarce life-sustaining technology to those imminently dying with or without the technology. There is no empirical or ethical justification for removing life support based on score-based algorithms without the permission of the patient or surrogate. Using such algorithms for the initial distribution of resources is difficult to justify when faced with novel pathogens where a priori survival predictions are poor. New York State has a precedent of allowing two physicians to withdraw or withhold life-sustaining treatment when there is no surrogate decision-maker available and the patient lacks capacity to make decisions. The criteria, however, are very narrow, including that the patient must be "imminently dying with or without the life-sustaining treatment" (Family Health Care Decisions Act 2010). This standard could be applied more broadly to patients with surrogates when crisis standards of care are implemented to avoid waste, upholding the duty to steward resources. Bioethics committees that include community members who are truly representative of the community should provide ongoing real-time review of such decisions during a disaster.

However, we cannot return to ad hoc bedside decisionmaking that was seen during Hurricane Katrina. The possibility will always exist of absolutely depleting all resources and forcing a choice [40]. If triage decisions remain unavoidable even after the above measures are taken, a lottery with a weighting system that takes into account social justice goals could be considered. Still, criteria that enter lottery weights must be very carefully considered to avoid ageism, ableism, and racism $[7 \bullet, 19 \bullet \bullet$. In addition, this approach is limited due to the fact that many patients who equity weights are intended to assist will be seen in minority-serving hospitals. Using these weights within one hospital may do little to offset structural inequalities if most patients are from similarly disadvantaged areas. Prioritizing such facilities for stockpiling of resources would be a better approach. If age is to be considered using the "fair innings" approach, every effort must be made to protect older adults from becoming ill in the first place, by prioritizing them first for PPE and other protective measures.
To be ethically permissible, triage strategies must be rooted in community values. Extensive community feedback on any new approach is needed. The current period is an ideal time to revisit community normative values around triage. Previous work with communities on this issue has employed hypothetical scenarios, but communities now have first-hand experiences of scarcity which may have clarified values and preference.

Third, the mental health of healthcare workers must be protected. Many articles and mainstream media have spoken about the third wave of COVID-19, resulting in the emergence of widespread mental health challenges, including burnout and mass exodus of our frontline medical workers. Health care workers have been heralded as heroes - yet most have failed to receive hazard pay, and many healthcare workers who perform the most essential and basic care duties exist on a wage that within many states keeps them at or below the Federal Poverty line [41].

There are many evidence-based methods to enhance healthcare professionals' ability to deal with and manage distressing situations associated with their work. These include mind-based programs targeted to increase resilience to more physical movement or direct telephone/crisis support [42-44]. Yet what seems to be missing from many of these programs is the impetus on a healthcare organization to rethink how it can restructure direct care services to quickly assess and address mental health concerns of its personnel without judgment or penalty. Until systems of care recognize and appreciate the human nature of healthcare work and leverage resources to ensure that the humanism of this work is valued, there will always be an increased risk for healthcare professionals to develop mental health issues throughout their career trajectory. An effective system would include multiple avenues for people to seek judgment-free help for their valid concerns.

Stress during disaster conditions sometimes results from moral injury, which occurs when an individual is forced to act in a way that transgresses their ethical standards. Debriefing with the clinical ethics consult team offers an approach that may more accessible to clinicians compared with traditional mental health care. First, it shifts the focus away from individual psychopathology and instead highlights the pathologic nature of the situation that led to the moral injury. Second, it allows for retrospective analysis of decisions using moral frameworks, which may help restore individuals' sense of moral worth, which can lead to better psychological flexibility and resilience.

Finally, the neglect and de-prioritization of LTCs in disaster planning and distribution of PPE illustrate the intersection between ageism, ableism, and racism in our society, given that LTC residents are vulnerable elders and those with disabilities and LTC workers are disproportionately 
from minority and marginalized communities. It was already known prior to COVID-19 that LTCs would be particularly vulnerable to novel infectious diseases. The lack of planning for this eventuality is unconscionable neglect of large segments of our society and must be rectified going forward.

\section{References}

\section{Papers of particular interest, published recently, have been highlighted as: \\ - Of importance \\ $\bullet$ Of major importance}

1. Crisis Standards of Care. A Systems Framework for Catastrophic Disaster Response. Mil Med. 2016;181(8):719-21.

2. New York State Taskforce on Life and the Law Ventilator Triage Guidelines: New York State Department of Health; 2015 [cited New York State Taskforce on Life and the Law. Available from: https:// www.health.ny.gov/regulations/task_force/reports_publications/ docs/ventilator_guidelines.pdf.

3. Daugherty Biddison EL, Gwon H, Schoch-Spana M, Cavalier R, White DB, Dawson T, et al. The community speaks: understanding ethical values in allocation of scarce lifesaving resources during disasters. Ann Am Thorac Soc. 2014;11(5):777-83.

4. Piscitello GM, Kapania EM, Miller WD, Rojas JC, Siegler M, Parker WF. Variation in Ventilator Allocation Guidelines by US State During the Coronavirus Disease 2019 Pandemic: A Systematic Review. JAMA network open. 2020;3(6):e2012606-e.

5. Mello MM, Persad G, White DB. Respecting Disability Rights - Toward Improved Crisis Standards of Care. The New England journal of medicine. 2020;383(5):e26. This ethical analysis called for the removal of categorical exclusions that preclude groups from accessing scarce life-sustaining resources that unfairly penalize people with chronic disease and disability.

6. Auriemma CL, Molinero AM, Houtrow AJ, Persad G, White DB, Halpern SD. Eliminating Categorical Exclusion Criteria in Crisis Standards of Care Frameworks. The American journal of bioethics : AJOB. 2020;20(7):28-36.

7. White DB, Lo B. Mitigating Inequities and Saving Lives with ICU Triage during the COVID-19 Pandemic. Am J Respir Crit Care Med. 2021;203(3):287-95. New triage guidelines adding "equity weights" to give some priority to disadvantaged groups, e.g. using the Area Deprivation Index, and prioritizing broadly-defined essential workers.

8. Ford CL, Airhihenbuwa CO. Critical Race Theory, race equity, and public health: toward antiracism praxis. Am J Public Health. 2010;100 Suppl 1(Suppl 1):S30-5.

9. Aulisio MP. Why Did Hospital Ethics Committees Emerge in the US? AMA J Ethics. 2016;18(5):546-53.

10. Bodzin AS, Baker TB. Liver Transplantation Today: Where We Are Now and Where We Are Going. Liver transplantation : official publication of the American Association for the Study of Liver Diseases and the International Liver Transplantation Society. 2018;24(10):1470-5.

11. Ladin K, Emerson J, Berry K, Butt Z, Gordon EJ, Daniels N, et al. Excluding patients from transplant due to social support: Results from a national survey of transplant providers. Am J Transplant Off J Am Soc Transplant Am Soc Transplant Surg. 2019;19(1):193-203.
12. Ladin K, Daniels A, Osani M, Bannuru RR. Is social support associated with post-transplant medication adherence and outcomes? A systematic review and meta-analysis. Transplant Rev (Orlando). 2018;32(1):16-28.

13. Harding K, Mersha TB, Webb FA, Vassalotti JA, Nicholas SB Current State and Future Trends to Optimize the Care of African Americans with End-Stage Renal Disease. Am J Nephrol. 2017;46(2):156-64.

14. Harding K, Mersha TB, Pham PT, Waterman AD, Webb FA, Vassalotti JA, et al. Health Disparities in Kidney Transplantation for African Americans. Am J Nephrol. 2017;46(2):165-75.

15. Fink S. Playing God [Internet]. New York, New York: WNYC Studios; 2016 August 21, 2016. Podcast. Available from: https:// www.wnycstudios.org/podcasts/radiolab/articles/playing-god

16. Curiel TJ. Murder or Mercy? Hurricane Katrina and the Need for Disaster Training. N Engl J Med. 2006;355(20):2067-9.

17. Braddock $\mathrm{CH}$ 3rd. Racism and Bioethics: The Myth of Color Blindness. The American journal of bioethics : AJOB. 2021;21(2):28-32.

18. Farquharson WH, Thornton CJ. Debate: Exposing the most serious infirmity - racism's impact on health in the era of COVID-19. Child Adolesc Mental Health. 2020;25(3):182-3.

19.• Schmidt H, Roberts DE, Eneanya ND. Rationing, racism and justice: advancing the debate around 'colourblind' COVID-19 ventilator allocation. J Med Ethics. 2021. This ethical analysis explored adding "equity weights" to give some priority to disadvantaged groups, e.g. using the Area Deprivation Index, and adjusting or eliminating SOFA score-based algorithms.

20. Stone JR. Social Justice, Triage, and COVID-19: Ignore Life-years Saved. Med Care. 2020;58(7):579-81.

21. Cuartas PA, Tavares Santos H, Levy BM, Gong MN, Powell T, Chuang E. How Useful Are Sequential Organ Failure Assessment (SOFA) Score-Based Ventilator Triage Guidelines During the COVID-19 Pandemic? TP11 TP011 use of health services and medical education research to evaluate the effect of COVID-19. Am Thoracic Soc Int Conf Abstracts: Am Thoracic Soc 2021;A1494-A.

22. Wunsch H, Hill AD, Bosch N, Adhikari NKJ, Rubenfeld G, Walkey A, et al. Comparison of 2 Triage Scoring Guidelines for Allocation of Mechanical Ventilators. JAMA network open. 2020;3(12):e2029250-e.

23. Jansen LA, Wall S. Weighted Lotteries and the Allocation of Scarce Medications for Covid-19. Hastings Cent Rep. 2021;51(1):39-46.

24. White DB, Lo B. A Framework for Rationing Ventilators and Critical Care Beds During the COVID-19 Pandemic. JAMA. 2020;323(18):1773-4.

25. Bowden E, Campanile C, Golding B. Worker at NYC hospital where nurses wear trash bags as protection dies from coronavirus: New York Post; 2020 [updated March 25, 2020. Available from: https://nypost.com/2020/03/25/worker-atnyc-hospital-where-nurses-wear-trash-bags-as-protectiondies-from-coronavirus/.

26. Livingston E, Desai A, Berkwits M. Sourcing Personal Protective Equipment During the COVID-19 Pandemic. JAMA. 2020;323(19):1912-4.

27. Berlinger N, Wynia M, Powell T, Hester DM, Milliken A, Fabi R, et al. Ethical Framework for Health Care Institutions Responding to Novel Coronaviros SARS-CoV-2 (COVID-19). Guidelines for Institutional Ethis Servies Responding to COVID-19: Managing Uncertainty, Safeguarding Commuinities, Guiding Practice. The Hastings Center; 2020 March 16, 2020.

28. Hughes MM, Groenewold MR, Lessem SE, Xu K, Ussery EN, Wiegand RE, et al. Update: Characteristics of Health Care Personnel with COVID-19 - United States, February 12-July 16, 2020. MMWR Morb Mortal Wkly Rep. 2020;69(38):1364-8. 
29. Kaiser Family Foundation. State Reports of Long-Term Care Facility Cases and Deaths Related to COVID-19. 2020 [Available from: https://www.kff.org/health-costs/issue-brief/state-data-andpolicy-actions-to-address-coronavirus/.

30. Musumeci M, Chidambaram P. Key Question About Nursing Home Regulation and Oversight in the Wake of COVID-19. 2020 $8 / 3 / 2020$.

31. White EM, Wetle TF, Reddy A, Baier RR. Front-line Nursing Home Staff Experiences During the COVID-19 Pandemic. J Am Med Dir Assoc. 2021;22(1):199-203.

32. Turner MC, Marshall SD. Can gendered personal protective equipment design account for high infection rates in female healthcare workers following intubation? Anaesthesia. 2021;76(1):132-3.

33. Yarrow E, Pagan V. 'Reflections on frontline medical work during Covid-19, and the embodiment of risk'. Gender, work, and organization. 2021; 28( S1): 537-548. https://doi.org/10.1111/ gwao. 12505.

34. Mihailescu M, Neiterman E. A scoping review of the literature on the current mental health status of physicians and physicians-intraining in North America. BMC Public Health. 2019;19(1):1363.

35. Munnangi S, Dupiton L, Boutin A, Angus LDG. Burnout, Perceived Stress, and Job Satisfaction Among Trauma Nurses at a Level I Safety-Net Trauma Center. Journal of trauma nursing : the official journal of the Society of Trauma Nurses. 2018;25(1):4-13.

36. Labrague LJ, De Los Santos JAA. COVID-19 anxiety among front-line nurses: Predictive role of organisational support, personal resilience and social support. J Nurs Manag. 2020;28(7):1653-61.

37. Kim KW, Lim HC, Park JH, Park SG, Park YJ, Cho HH. Developing a Basic Scale for Workers' Psychological Burden from the
Perspective of Occupational Safety and Health. Saf Health Work. 2018;9(2):224-31.

38. Martins PN, Mustian MN, MacLennan PA, Ortiz JA, Akoad M, Caicedo JC, et al. Impact of the new kidney allocation system A2/ $\mathrm{A} 2 \mathrm{~B} \rightarrow \mathrm{B}$ policy on access to transplantation among minority candidates. Am J Transplant Off J Am Soc Transplant Am Soc Transplant Surg. 2018;18(8):1947-53.

39. Back AL, Grant MS, McCabe PJ. Public Messaging for Serious Illness Care in the Age of Coronavirus Disease: Cutting through Misconceptions, Mixed Feelings, and Distrust. J Palliat Med. 2021.

40. Rosenbaum L. Facing Covid-19 in Italy-Ethics, Logistics, and Therapeutics on the Epidemic's Front Line. NEJM. 2020;382(20):1873-5.

41. Byock I. Heroism and Hypocrisy: Seeing Our Reflection with 2020 Vision. J Palliat Med. 2020;23(11):1417-8.

42. Blake H, Bermingham F, Johnson G, Tabner A. Mitigating the Psychological Impact of COVID-19 on Healthcare Workers: A Digital Learning Package. Int j environ res pub health. 2020;17(9).

43. Jensen A, Bonde LO. The use of arts interventions for mental health and wellbeing in health settings. Perspect Public Health. 2018;138(4):209-14.

44. Gray P, Senabe S, Naicker N, Kgalamono S, Yassi A, Spiegel JM. Workplace-Based Organizational Interventions Promoting Mental Health and Happiness among Healthcare Workers: A Realist Review. Int j environ res pub health. 2019;16(22).

Publisher's Note Springer Nature remains neutral with regard to jurisdictional claims in published maps and institutional affiliations. 